\title{
The diagnosis of the scars of chronic pyelonephritis
}

\author{
J. F. SMITH \\ From the Department of Morbid Anatomy, University College Hospital \\ Medical School, London
}

SYNOPSIS The thesis put forward by Hodson (1959) from radiological evidence that renal scars due to ischaemia show a narrowed parenchyma associated with a normal calyceal pattern, whereas in $\vec{G}$ focal pyelonephritic scars the narrowed parenchyma is associated with clubbing of the calyx in the or affected area, has been investigated anatomically. The thesis finds considerable support and the $\underset{N}{N}$ importance of careful examination of the calyceal system in scarred kidneys is stressed. In only one 을 of 23 examples in which all the circumstantial evidence of the case pointed to an ischaemic scar was there any deformation of the renal calyx. In 13 of 14 examples in which the evidence pointed to $z$ chronic pyelonephritis there was clubbing of the calyx associated with the scar.

In chronic pyelonephritis the kidneys are usually unequal in size and show multiple irregular surface scars. Somewhat similar surface scars may result from purely ischaemic processes and it is accepted that ischaemic factors may play some part in chronic pyelonephritis. Weiss and Parker (1939) described the scars of benign nephrosclerosis as V-shaped and those of healed or chronic pyelonephritis as $U$ shaped. They admitted that scars of healed infarcts were similar to those of chronic pyelonephritis, and that grossly the two could be confused, but maintained that histologically there was rarely any difficulty in distinguishing them. Kimmelstiel (1960) has recently indicated the limitations of the classical histological criteria of chronic pyelonephritis. He has made explicit, what many have believed, that such changes as interstitial infiltration, dilated tubules with colloid casts, and vascular changes could be found in both ischaemic and chronic pyelonephritic scars.

Hodson (1959) claimed that vascular and pyelonephritic scars could be distinguished on radiological examination. He emphasized that in an ischaemic scar a narrowed renal parenchyma was associated with a normal calyceal pattern, whereas in a focal pyelonephritic scar the narrowed parenchyma was associated with clubbing of the calyx in the affected area. He presented no pathological evidence to support this contention, which appeared reasonable from knowledge of the pathogenesis of pyelonephritis, in particular its association with anatomical or functional obstruction in the lower urinary tract,

Received for publication 14 March 1962. and the fact that focal infection can cause focal $\overrightarrow{\mathscr{\theta}}$ destruction of tissue with subsequent scar formation. $\mathrm{N}$ This investigation was undertaken to provide pathological confirmation of Hodson's claim.

\section{METHODS}

Kidneys from necropsies and removed at operation were used. The position and size of surface scars, the size of $\overrightarrow{\overrightarrow{0}}$ the kidney, and the size and shape of the pelvis and all 3 calyces were carefully noted by ordinary anatomical methods. A most important point was the careful examination of all calyces. If the pelvis is of normal size it is easy to overlook a deformed calyx lying well outside the median plane of the kidney if a saggittal midline section $\overline{-}$ is made. In a few examples radiographs were made after $\underline{3}$. injection of an opaque material into the pelvicalyceal $\varnothing$ system via the ureter. The normal histological sections were made.

\section{CRITERIA FOR DIAGNOSIS}

Circumstantial evidence, as outlined below, for an $N$ ischaemic or pyelonephritic process was usually $N$ clear. The histological changes were also made use of, bearing in mind the limitations indicated by Kimmelstiel (1960). Only those cases in which with this evidence it was thought possible to put the $\stackrel{\varrho}{=}$ kidney firmly into one of the two groups were used $\mathscr{\Phi}$ in the investigation.

Circumstantial evidence for an ischaemic scar was the presence of an occlusive lesion of the renal artery $\frac{\pi}{\mathbb{D}}$ or one of its main branches or evidence suggestive $\frac{?}{\mathbb{P}}$ of an embolic process. This included, in postmortem $\varnothing$ cases, the presence of heart disease in which ante- $\underset{\gamma}{0}$ 
mortem thrombus was present in the left auricle or ventricle or could have been at some time; in some examples this was reinforced by the presence of scars of old infarcts in the brain or spleen, of embolic origin.

Circumstantial evidence for a chronic pyelonephritic scar was the presence of an anatomical or functional obstruction in the lower urinary tract. This included anomalies such as double ureter and the demonstration of vesico-ureteric reflux during life. In a few instances the history of a previous acute attack of pyelonephritis was available and accepted as evidence.

Examples where circumstantial evidence for both processes was present were excluded. In such it is possible that ischaemic and pyelonephritic scars were present in the same kidney. One case is included in which a pyelonephritic scar was present in the left kidney and a vascular scar in the right. Full use of histological material was made. This was found to be clearly in favour of one of the processes in most examples but not in all. Details of the histology are not discussed in this paper.

\section{ANALYSIS}

Thirty-seven kidneys were found suitable for comparison. Of these, 23 were assigned to the vascular group and 14 to the pyelonephritic. Of the $23 \mathrm{kid}$ neys with purely ischaemic scars, 19 were from necropsies and four were surgical specimens removed from patients with hypertension associated with unilateral renal disease. Four of the 14 pyelonephritic kidneys were obtained at necropsy. In seven of the 10 which were surgical specimens, the underlying aetiological factor was vesico-ureteric reflux. All the cases were examined by me personally and nearly all were collected in 1960 and 1961 ; a few preserved from 1959 in which full examination was possible were also used. (Several other examples of pyelonephritic scars associated with gross hydronephrosis or pyonephrosis were rejected from the series because such kidneys are not diagnostic problems; in none of these was the result in conflict with the main series.) Of the 23 assigned to the vascular group, 22 had no deformity of the renal pelvis or calyx, and one showed slight clubbing of the calyx associated with the scar. Of the 14 assigned to the pyelonephritic group, 13 had clubbed calyces associated with the surface scars; in 10 the pelvis was of normal size, in three it was slightly dilated. The one kidney with no calyceal deformity was from a diabetic with clear clinical evidence of unilateral acute pyelonephritis in the scarred kidney a few years before death.

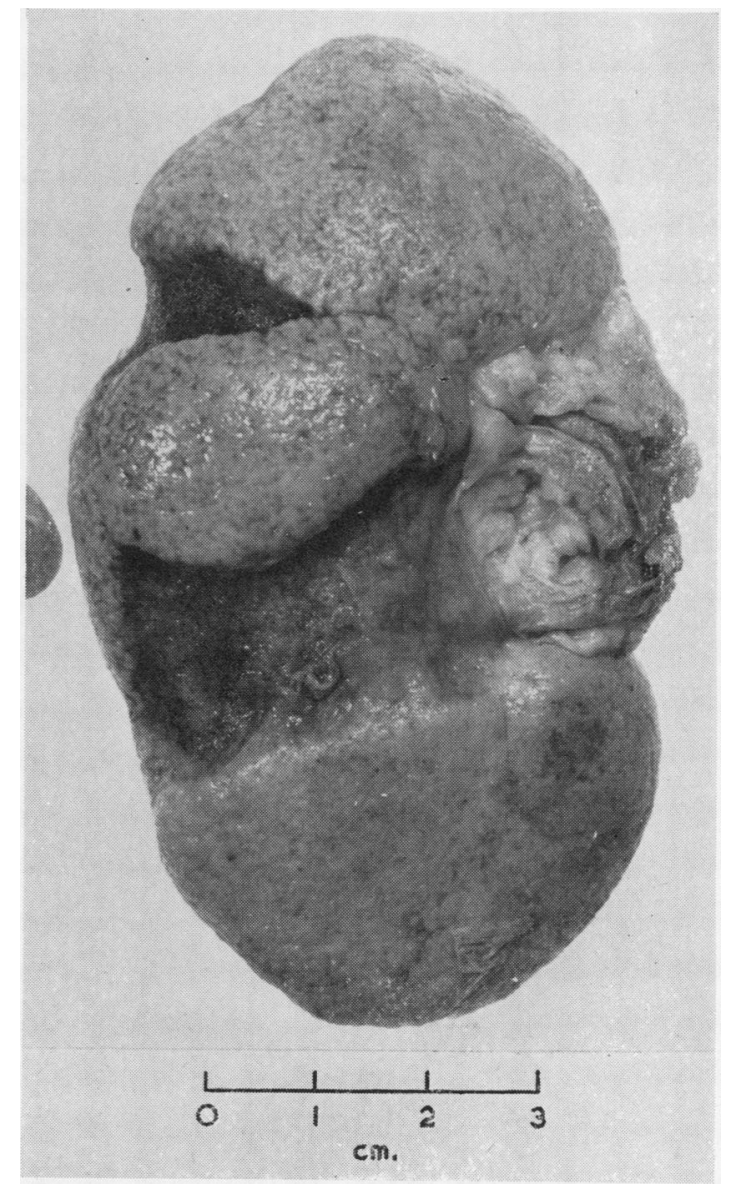

FIG. 1. Broad scars on surface of a kidney from an 83-year-old man. Circumstantial and histological evidence in this case favours a vascular aetiolog.

CASE REPORTS

ISCHAEMIC SCARS 1 An 83-year-old man with old and recent myocardial infarction due to coronary thrombosis had two broad, deep scars on the anterior surface of the left kidney (Fig. 1). Neither the pelvis nor calyces were deformed (Fig. 2). Microscopical examination showed changes typical of old infarction with partial or complete occlusion of arcuate and interlobular arteries in the scars.

2 A 53-year-old woman with mitral stenosis and antemortem thrombus on the wall of the left auricle with evidence of old infarction in the brain had one large scar on the surface of each kidney together with a few smaller ones. No deformity of the calyces was found on injection of opaque material via the ureter or on dissection. Microscopical examination showed old infarction with organized thrombo-embolus in one vessel (Fig. 3).

PYELONEPHRITIC SCARS 1 A 10-year-old girl had a sixyear history of recurrent debilitating urinary infections. 


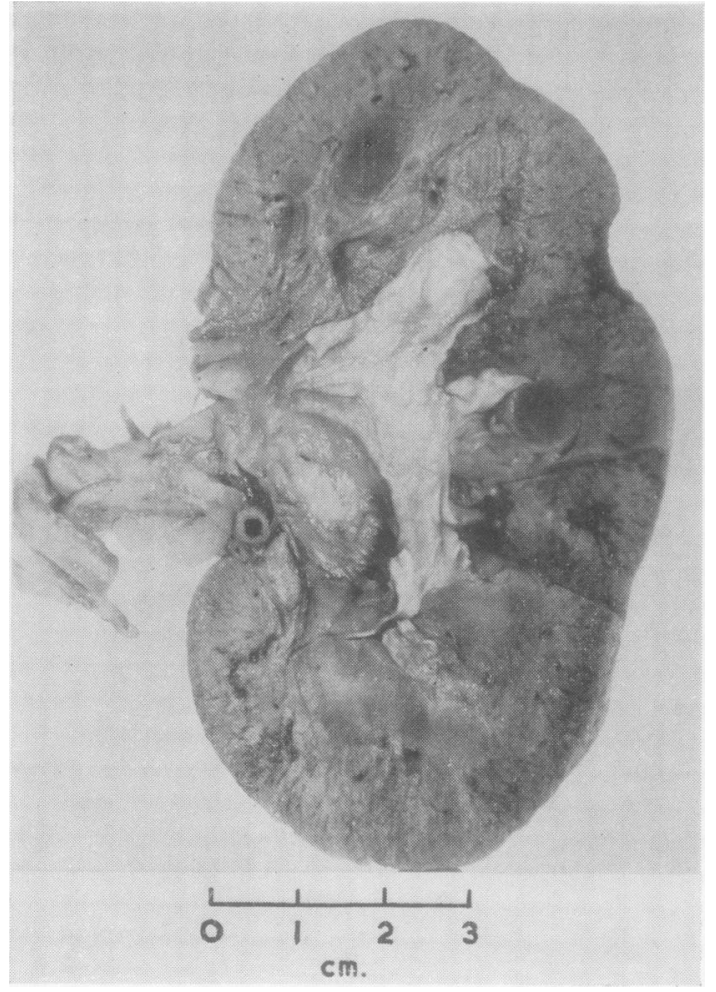

FIG. 2

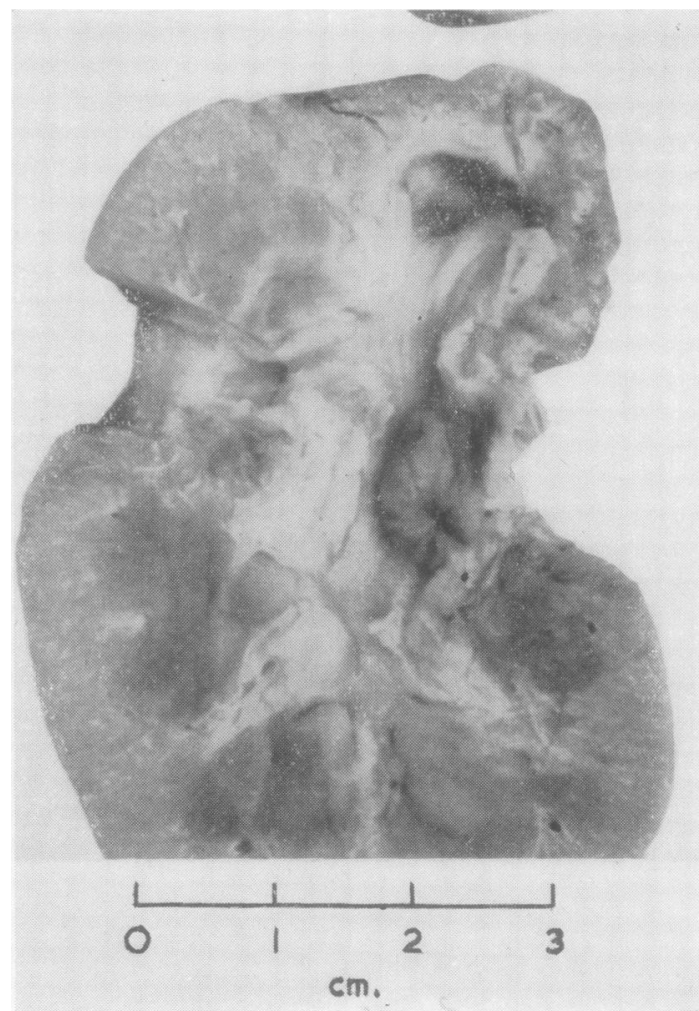

FIG. 4

FIG. 2. Cut surface of kidney depicted in Fig. 1; no deformity of calyces related to scars.

FIG. 4. Pyelonephritic scars in the kidney of a 10-year-old girl. Note the clubbed calyx at the upper pole, the adjacent papilla being normal and the next calyx clubbed but out of the median plane of section.

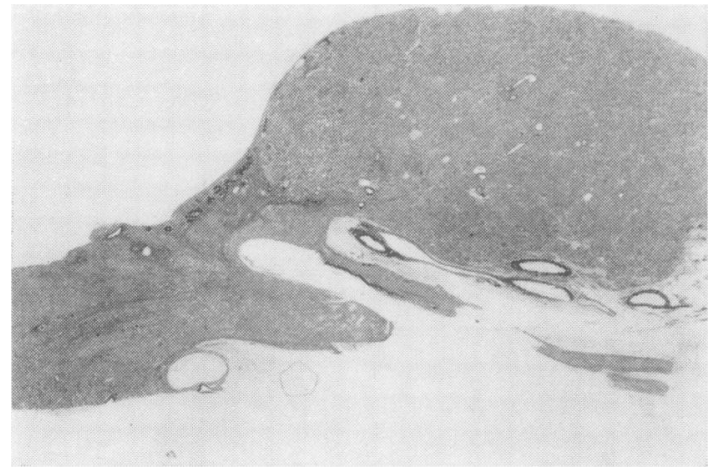

FIG. 3. Section of old infarct in the kidney of a 53-year-old woman with mitral stenosis, thrombus in the left auricle, and multiple emboli (van Gieson and elastic, $\times 2.7$ ). Note a preserved papilla beneath the scar of the infarct.
Radiological examination showed an area of scarring at 8 the upper pole of the right kidney when she was $5 ₹$ years old, and a further area affecting the lowest of the 음 three pyramids of the upper pole when she was 10 years old but sparing the middle one. Micturating cystography showed a normal bladder and urethra with reflux up the right ureter into the renal pelvis. The right kidney was $\mathrm{N}$ removed. It was shrunken $(7 \times 4.5 \times 2.5 \mathrm{~cm}$.) and showed a marked depressed scar on the surface of the upper pole 0 merging into another on the posterior wall but the two $\mathrm{\omega}$ were separated by smooth cortex at the lateral border. On section there was gross narrowing of the parenchyma 0 at the site of these two scars with clubbing of the calyces $\frac{\Gamma}{\Phi}$ (Fig. 4). The two so affected were separated by a normal pyramid corresponding to the smooth surface area. The lower clubbed calyx was well out of the median saggittal $\bar{D}$ plane of the kidney and required careful nibbling away $\frac{\vec{D}}{\mathbb{D}}$ of parenchyma to expose it. Microscopical examination $\stackrel{?}{+}$

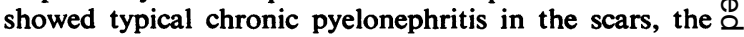
vascular involvement being rather scanty. The pelvic and 


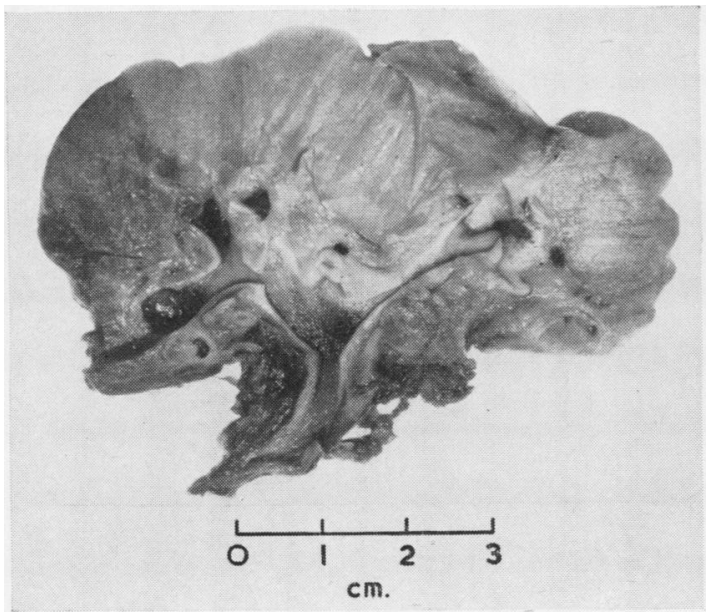

FIG. 5. Pyelonephritic scars with clubbed calyces in the kidney of a 38-year-old woman. The renal pelvis is not dilated.

calyceal mucosa was chronically inflamed. The parenchyma between the scars showed no abnormality.

2 A 38-year-old woman was found to have hypertension on routine examination. She had had 'pyelitis' of pregnancy at the age of 20 . Radiological investigation showed an abnormality at the upper pole of the right kidney which was smaller than the left. Following further investigations, including a negative examination for vesico-ureteric reflux, this kidney was excised. It showed numerous coarse depressions and a few small cysts on the outer surface. Figures 5 and 6 illustrate the clubbed calyces associated with the surface scars, the necessity for examining out of the median sagittal plane of the initial section of the kidney, and the normal size of the renal pelvis. Histological examination showed typical chronic pyelonephritis in the scars and chronic inflammation of pelvic and calyceal mucosa.

\section{DISCUSSION}

This investigation indicates that it is worth while paying careful attention to the renal calyces in attempting a differential diagnosis of aetiology in an irregularly scarred kidney. Deformity of the calyx corresponding to a surface scar is far more likely to indicate a pyelonephritic than a vascular process. The absence of deformity does not necessarily exclude pyelonephritis but it suggests that very careful consideration should be given to the possibility of a primary ischaemic process.

Some of the earlier writers on chronic pyelonephritis had mentioned deformities or dilatations of the renal calyces in individual cases (Gibson, 1928; Longcope and Winkenwerder, 1933). Staemmler and

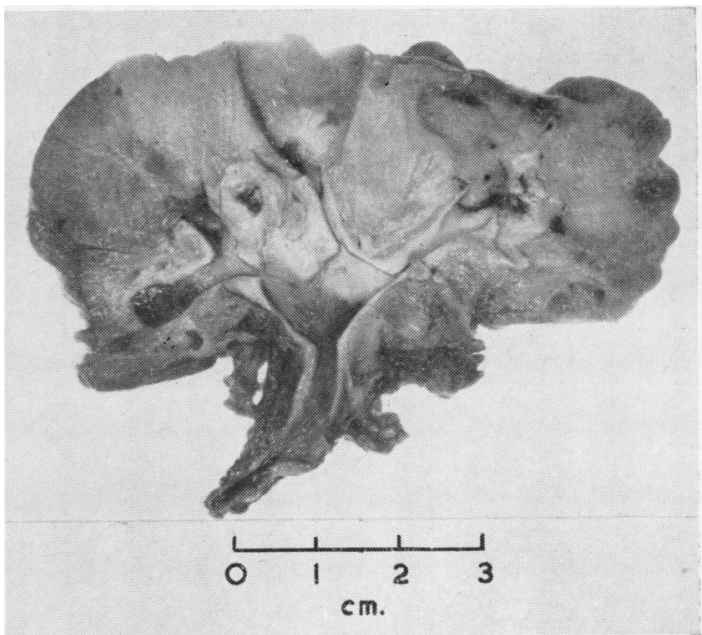

FIG. 6. A wedge of tissue removed from the kidney shown in Fig. 5, showing a further clubbed calyx out of the median plane of section.

Dopheide (1930) had referred to dilatation of the pelvis and especially of the calyx as a characteristic macroscopic feature of the disease. However, the deformed calyx is seldom referred to in textbooks of pathology at the present time. The reviews by Kleeman, Hewitt, and Guze (1960) and by Gall (1961) pay little attention to it, and it is scarcely considered in the series of papers relevant to anatomical factors in 'The Biology of Pyelonephritis' (Kimmelstiel, 1960). The present study, although of limited scope, does support Hodson's (1959) thesis. Thus in only one of 23 examples in which all the circumstantial evidence of the case pointed to an ischaemic aetiology for the scar was there any deformation of the renal calyx. In 13 of the 14 examples in which the evidence pointed to chronic pyelonephritis there was clubbing of the calyx associated with the scar.

Two things emerged from the investigation which were surprising. One was how easy it is to neglect the careful examination of calyces well out of the median sagittal plane of the kidney. The other was that in so many of the pyelonephritic kidneys the renal pelvis was of normal size although the calyces were grossly deformed. This is probably because of selection of material. An unusually high proportion of the cases in this series were examples of pyelonephritis in young subjects associated with reflux. It served the purpose, however, of focusing attention on kidneys in which the diagnosis was not immediately obvious rather than on the group with marked evidence of generalized back pressure, e.g, 
the infected hydronephrotic kidneys following marked prostatic enlargement.

The thesis presented in this paper depends on the acceptance of the view that the vast majority of cases of pyelonephritis are associated with some predisposing obstructive factor in the urinary tract. Most pathologists and clinicians accept this but a few do not (De Wardener, 1961).

I am grateful to Sir Roy Cameron, F.R.S., for advice on the manuscript.

\section{REFERENCES}

De Wardener, H. E. (1961). Brit. med. J., 1, 1235.

Gall, E. A. (1961). Bull. N. Y. Acad. Med., 37, 367.

Gibson, A. G. (1928). Lancet, 2, 903.

Hodson, C. J. (1959). Proc. roy. Soc. Med., 52, 669.

Kimmelstiel, P. (1960). Significance of Chronic Pyelonephritis. In The Biology of Pyelonephritis, ed. E. L. Quinn and E. H. Kass. Churchill, London.

Kleeman, C. R., Hewitt, W. L., and Guze, L. B. (1960). Medicine (Baltimore), 39, 3.

Longcope, W. T., and Winkenwerder, W. L. (1933). Bull. Johns Hopk. Hosp., 53, 255.

Staemmler, M., and Dopheide, W. (1930). Virchows Arch. path. Anat., 277, 713.

Weiss, S., and Parker, F. (1939). Medicine (Baltimore), 18, 221.

\section{Broadsheets prepared by the Association of Clinical Pathologists}

The following broadsheets (new series) are published by the Association of Clinical Pathologists. They may be obtained from Dr. R. B. H. Tierney, Pathological Laboratory, Boutport Street, Barnstaple, N. Devon. The prices include postage, but airmail will be charged extra.

3 The Detection of Barbiturates in Blood, Cerebrospinal Fluid, Urine, and Stomach Contents. 1953. L. c. NICKOLLS. 1s.

4 The Estimation of Carbon Monoxide in Blood. 1953. D. A. STANLEY. $1 \mathrm{~s}$.

5 The Identification of Reducing Substances in Urine by Partition Chromatography on Paper. 1953. G. B. MANNING. $1 \mathrm{~s}$.

6 The Paul-Bunnell Test. 1954. R. H. A. SWAIN. 1s.

7 The Papanicolaou Technique for the Detection of Malignant Cells in Sputum. 1955. F. HAMPSON. 1s.

10 Mycological Techniques: (1) Collection of Specimens. 1956. R. W. RIDDELL. $1 \mathrm{~s}$.

11 Mycological Techniques: (2) Cultural Isolation. 1956. R. W. RIDDELL. 1s.

12 Techniques for Demonstrating L.E. Cells. 1956. J. v. DACIE and L. S. SACKER. 1s.

13 The Identification of Serotypes of Escherichia coli Associated with Infantile Gastro-enteritis. 1956. JOAN TAYLOR. 1s.

14 The Determination of Serum Iron and Serum Unsaturated Iron-binding Capacity. 1956. ARTHUR JORDAN. 1s.

16 Preservation of Pathological Museum Specimens. 1957. L. W. PROGER. 1s.

17 Cultural Diagnosis of Whooping-cough. 1957. B. W. LACEY. $1 \mathrm{~s}$.

18 The Rose-Waaler Test. 1957. C. L. GREENBURY. 1s.

20 Investigation of Porphyrin/Porphyria. 1958 (reprinted 1962). C. RIMINGTON. 2s.

23 The Dried Disc Technique for Bacterial Sensitivity Tests. 1959. R. W. FAIRBROTHER and J. C. SHERRIS. 1s.
24 Safe Handling of Radioactive Tissues in the Laboratory and Post-mortem Room. 1959. R. C. CURRAN. 1s.

26 The Periodic Acid-Schiff Reaction. 1959. A. G. E. PEARSE $1 \mathrm{~s}$.

28 Daily Fatty Acid Excretion. 1960. A. C. FRAZER. 2s.

29 The Preparation of Bone for Diagnostic Histology. 1960. D. H. Collins. 2s.

30 Control of Accuracy in Chemical Pathology. 1961. G. H. GRANT. 4s.

31 Investigation of Haemorrhagic States with Special Reference to Defects of Coagulation of the Blood. 1961. E. K. BLACKBURN. 4s.

32 Detection of Resistance to Streptomycin, P.A.S., and Isoniazid in Tubercle Bacilli. 1961. R. CRUICKSHANK and S. M. STEWART. $2 \mathrm{~s}$.

33 The Laboratory Detection of Abnormal Haemoglobins. 1961. H. LEHMANN and J. A. M. AGER. 4s.

34 Titration of Antistreptolysin O. 1961. H. GOODER and R. E. O. WILLIAMS. $2 \mathrm{~s}$.

35 The Estimation of Faecal 'Urobilinogen'. 1961. C. H. GRAY. 2s.

36 Quantitative Determination of Porphobilinogen and Porphyrins in Urine and Faeces. 1961. C. RIMINGTON. 3s. 6d.

37 The Paper Electrophoresis of Serum and Urinary Proteins. 1961. G. FRANGLEN and N. H. MARTIN. 4s.

38 The Augmented Histamine Gastric Function Test. 1961. M. LUBRAN. 2s.

39 Investigation of Haemolytic Anaemia. 1961. J. G. SELWYN. $2 \mathrm{~s}$.

40 Short Term Preservation of Bacterial Cultures. 1962. E. Joan Stokes. 2s. 